\title{
A novel, streamline-based injection efficiency enhancement method using multi-objective genetic algorithm
}

\author{
Mohammad Amin Safarzadeh • Mehdi Motealleh • \\ Jamshid Moghadasi
}

Received: 9 January 2013 / Accepted: 5 May 2014/Published online: 14 June 2014

(C) The Author(s) 2014. This article is published with open access at Springerlink.com

\begin{abstract}
Water flooding is one of the most important techniques of improved oil recovery. However, two major problems, loss of injected water to the aquifer and unwanted water production, reduce the efficiency of water flooding. An improper allocation of water to injection wells usually is the main reason of these problems. In this paper, both multi-objective genetic algorithm (MOGA) optimization, which is more rapid and accurate than conventional $\mathrm{GA}$, and streamline simulation with the unique advantages of determination of flow path and participation of each injection well in total field oil production, were used in order to appease the mentioned problems. All previous studies have tried to optimize the injection rates based on injection efficiencies that were defined with the application of streamline simulation, while using MOGA optimization and Pareto concept always let us select the best global solutions with regard to the other defined criterions. Final solution in MOGA optimization is a set of correct answers. So, five scenarios, including different restrictions and economic situations were introduced. Best solution was obtained and compared with the common method of water injection optimization exclusively based on improving the efficiencies of injection wells. Results show that MOGA optimization always offers the best solution and all MOGA
\end{abstract}

\footnotetext{
M. A. Safarzadeh

Research Institute of Petroleum Industry, Tehran, Iran e-mail: safarzadehma@ripi.ir

M. Motealleh

Computer Aided Process Engineering Group, Tehran, Iran

e-mail: m.motealleh@capegroup.ir

J. Moghadasi ( $\square)$

Petroleum University of Technology, Ahwaz, Iran

e-mail: J.moghadasi@put.ac.ir
}

scenarios have better proficiencies than common optimization methods. Comparison of the proposed methodology in this paper with conventional workflow shows that MOGA, in the best case, can increase total oil production up to $6.5 \%$, and after considering all limitations, it can increase total oil production up to $4 \%$ and decrease the loss of water to the aquifer to about $26 \%$ in comparison with the common workflows.

Keywords Injection efficiency - Genetic algorithm · Multi-objective optimization - Streamline simulation . Pareto front · Waterflooding

\section{Introduction}

Water flooding an oil field has been a widely accepted method for increasing a reservoir's recovery since 1950 s. Possible problems associated with waterflood techniques due to unsuitable conditions are destructive effect on fluid transport within the reservoir and early water breakthrough. Therefore, knowing the allocation of flow between well pairs is the starting point of any technique to balance well patterns in waterflood process. Conventionally, allocation numbers have been determined using sophisticated empirical methodologies and then replaced by an immediate and rigorous streamlines solution method (Thiele 2001).

An important component in optimizing field performance is to be able to compare and rank the efficiency of injectors. One of the useful information provided by the streamlines is well allocation factors (WAF). This quantifies the amount of flow in a particular well due to other wells in the system. Since streamlines start at a source and end in a sink, it is possible to determine which injectors (or part of an aquifer) are supporting a particular producer and 
vice versa (Thiele 2001). Instead of moving fluids from cell-to-cell, streamline simulation breaks up the reservoir into one-dimensional systems, or tubes. The transport equations are then solved along the streamlines using the concept of time-of-flight. Decoupling the transport problem from the underlying 3D geological model results in a significant computational efficiency while minimizing numerical diffusion and grid orientation effects (Thiele et al. 1997; Samier et al. 2001).

\section{Waterflooding optimization}

Thiele and Batycky (2006) proposed an approach to predict well rate targets of injectors and producers to improve waterflood management (Appendix). Their approach is based on streamline simulation and the injection efficiency for injector-producer pairs. Assuming the fixed well status in mature fields with a constraint of total available water, Thiele and Batycky demonstrated improved waterfloods by reallocating injection water from low-efficiency to highefficiency injectors. The methodology was also implemented in naturally fractured reservoirs (2007) by Iino and Arihara (2007). Alhuthali et al. (2007) proposed a practical approach, based on equalizing the arrival times of the waterflood front at all producers for computing optimal injection and production rates. Almost all of the previous studies were based on optimizing the current injection efficiencies without any knowledge of initial rates while in this study, the injection rates are allocated in the field initially in the best way by the means of multi-objective genetic algorithm.

\section{Multi-objective genetic algorithm optimization}

Multi-objective formulations are realistic models for many complex engineering optimization problems. In many real-world problems, objectives under consideration conflict with each other, and optimizing a particular solution with respect to a single objective can result in unacceptable results with respect to the other objectives (Konak et al. 2006). Genetic algorithms are global optimization techniques, which means they converge to the global solution rather than to a local solution. The specific mechanics of the algorithms involve the language of microbiology and, in developing new potential solutions, mimic genetic operations (Marler et al. 2004). A general multi-objective optimization problem can be described as a vector function $f$ that maps a tuple of $m$ parameters (decision variables) to a tuple of $n$ objectives. Formally:

$$
\begin{aligned}
\operatorname{Min} / \operatorname{Max} y= & f(x)=\left(f_{1}(x), \ldots, f_{n}(x)\right) \\
\text { subject to } x= & \left(x_{1}, x_{2}, \ldots, x_{m}\right) \in \mathrm{X}, y=\left(y_{1}, y_{2}, \ldots, y_{m}\right) \\
& \in Y
\end{aligned}
$$

where $x$ is called the decision vector, $X$ is the parameter space, $y$ is the objective vector, and $Y$ is the objective space. After the first pioneering work on multi-objective evolutionary optimization in the 1980s (Schaffner 1984, 1985), several different algorithms have been proposed and successfully applied to various problems. Zitzler puts forward the Strength Pareto EA (SPEA) which had been applied it to the multi-objective problem successfully (Xie et al. 2005).

Classical multi-objective optimization methods transform multi-objective functions into a single objective function through the evaluation function which has some disadvantages such as essential need for providing weights, non-uniform distributed optimal solution due to local optimal searching algorithm and to repeatedly search algorithm for alternative solution (Xie et al. 2005).

The other general approach in multi-objective optimization is to determine the entire Pareto-optimal solution sets which are often preferred to a single solution, because they can be practical when considering real-life problems (Konak et al. 2006). They give the engineers the option to assess the trade-offs between different designs (Xie et al. 2005; Sbalzariniy et al. 2000). Pareto-optimal sets can be of varied sizes, but the size of the Pareto set usually increases with the increase in the number of objectives (Iino and Arihara 2007).

As GA requires little knowledge about the problem being solved, and they are easy to implement, robust, and inherently parallel, they can be effective regardless of the nature of the objective functions and constraints (Marler et al. 2004; Sbalzariniy et al. 2000). Being a population-based approach, multi-objective GA is well suited to find Pareto-optimal front. The ability of GA to simultaneously search different regions of a solution space makes it possible to find a diverse set of solutions for difficult problems with non-convex, discontinuous, and multi-modal solutions spaces. The crossover operator of GA may exploit structures of good solutions with respect to different objectives to create new non-dominated solutions in unexplored parts of the Pareto front. In addition, most multi-objective GA does not require the user to prioritize, scale, or weigh objectives. Therefore, GA has been the most popular heuristic approach to multi-objective design and optimization problems (Konak et al. 2006).

\section{Methodology}

Multi-objective genetic algorithm optimization of injection rate determination procedure was preceded according to the following workflow: 
Table 1 Multi-objective GA parameter values used in waterflooding optimization

\begin{tabular}{lllll}
\hline $\begin{array}{l}\text { Crossover } \\
\text { fraction }\end{array}$ & $\begin{array}{l}\text { Migration } \\
\text { fraction }\end{array}$ & $\begin{array}{l}\text { Pareto front } \\
\text { fraction }\end{array}$ & $\begin{array}{l}\text { Maximum } \\
\text { generation }\end{array}$ & $\begin{array}{l}\text { Function } \\
\text { tolerance }\end{array}$ \\
\hline 0.8 & 0.2 & 0.34 & 100 & $10^{-6}$ \\
\hline
\end{tabular}

1. Hundred sets of well water injection rates are constructed by GA (genetic algorithm) as an initial random population. For the considered population, injection efficiencies of all wells in each set are calculated using the following equation by means of the streamline simulation as the initial population cost in the GA calculations. Streamline simulation is used to calculate offset oil production rate of each injection well in all sets.

$\mathrm{IE}=\frac{\text { offset oil production rate }}{\text { water injection rate }}$

Therefore, in this case injection efficiencies would be defined as follows:

$\mathrm{IE}_{1}=\frac{\sum_{i=1}^{i=5} \text { offset oil production rate of } \mathrm{P}_{i} \text { due to } \mathrm{I}_{1}}{\text { water injection rate of } \mathrm{I}_{1}}$

$\mathrm{IE}_{2}=\frac{\sum_{i=1}^{i=5} \text { offset oil production rate of } \mathrm{P}_{i} \text { due to } \mathrm{I}_{2}}{\text { water injection rate of } \mathrm{I}_{2}}$

2. Population breeding process is implemented by defining the objective functions as simultaneous maximization of the population cost. This maximization process begins by non-dominated sort of chromosomes and then tournament selection of them. Multi-objective GA parameter are shown in Table 1.

3. In all scenarios, the final population is obtained around 50 generations when the function tolerance limit was reached. The best sets of answers for the objective functions (simultaneous maximization of injection efficiencies) are chosen by the means of Pareto solution set. Members of this improved generation are the most suitable sets of well water injection rates.

4. Although all Pareto-optimal solutions are the correct answers of objective functions, decision-making of the final optimum set must be implemented directly based on the desired criteria. Therefore, various scenarios are presented in this study. These scenarios are generated with consideration of different economic conditions, water production limitations and fluid loss to the aquifer. Any of these scenarios can be used for different approaches of field management and preservation.
Scenario A Minimum distance to average efficiency. The best answer can be the one with the minimum difference between wells injection efficiencies and average injection efficiency. In two-objective function Pareto algorithm solution sets, the best answer could be the nearest answer to the line $\operatorname{Eff}_{\text {well1 }}=\operatorname{Eff}_{\text {well2 }}$.

In this case, the selected injection efficiencies are the most balanced efficiencies.

Scenario $B$ Highest oil production. The best answer can be the one with the highest oil production rate set in Pareto solutions.

Scenario $C$ Lowest water production scenario. The best answer can be the one with the lowest water-cut set in Pareto solutions.

Scenario $D$ Lowest loss water. The best answer can be the one with the lowest edge water loss to the aquifer set in Pareto solutions.

Scenario $E$ Highest defined optimizing parameter scenario. The best answer can be the one with the maximum defined optimizing parameter set in Pareto solutions:

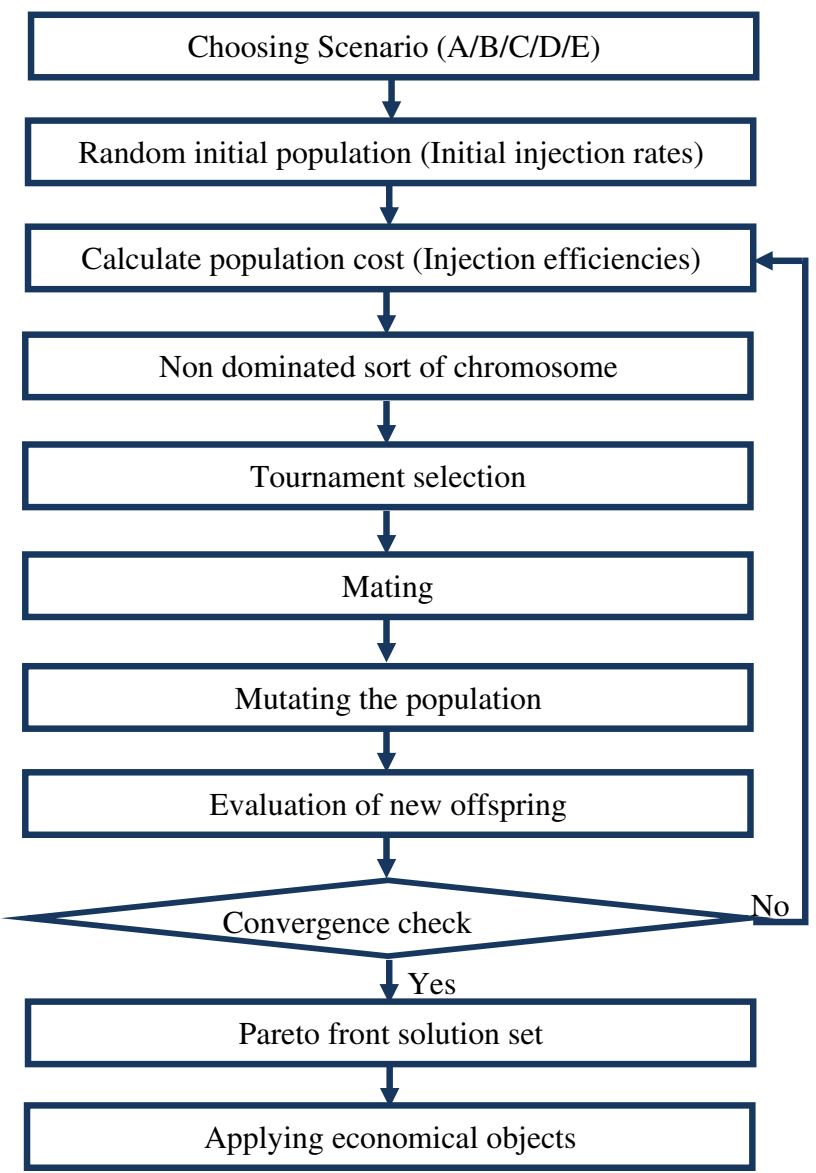

Fig. 1 Workflow diagram of a GA multi-objective optimization process 
Defined optimizing parameter

$$
=\left(\frac{\text { Oil production rate }}{\text { Water cut } \times \text { Edge fluid loss }}\right)
$$

5. The final reservoir condition at the end of the first time step will be generated by streamline simulation of the best answer determined from one of the previous section scenarios.

6. To obtain the most suitable allocation of well water injection rates, considering constant field water injection rate for the following time steps, sections 1-5 are repeated. Workflow diagram of a GA multi-objective optimization process is shown in Fig. 1.

\section{Synthetic model description}

Synthetic model, with two injectors and five producers was used to implement different multi-objective GA scenarios

Table 2 Reservoir rock and fluid properties

\begin{tabular}{ll}
\hline Properties & Value \\
\hline Dimension & $20 \times 20 \times 4$ \\
Cell size $(\mathrm{Dx} \times \mathrm{Dy} \times \mathrm{Dz})(\mathrm{ft})$ & $600 \times 600 \times 120$ \\
Initial water saturation $(\%)$ & 17 \\
Initial reservoir pressure (Psig) & 4,000 \\
Average porosity $(\%)$ & 14.5 \\
Average permeability $(\mathrm{md})$ & 25 \\
Water density $\left(\mathrm{Lb} / \mathrm{ft}^{3}\right)$ & 62.50 \\
Oil density $\left(\mathrm{Lb} / \mathrm{ft}^{3}\right)$ & 50.0 \\
Water viscosity $(\mathrm{Cp})$ & 1.0 \\
Oil viscosity $(\mathrm{Cp})$ & 0.82 \\
Water compressibility $\left(\mathrm{Psi}^{-1}\right)$ & $4.00 \mathrm{e}-006$ \\
Rock compressibility $\left(\mathrm{Psi}^{-1}\right)$ & $5.68 \mathrm{E}-06$ \\
\hline
\end{tabular}

and compared with conventional optimization workflow. The average reservoir rock and fluid properties are shown in Table 2. In order to consider well location heterogeneity, wells were located with specified different values of well spacing between 1,000 ft in I2-P3 and 5,000 ft in I1-P1.

Figure 2 shows well locations, oil saturation distribution and producers/injectors relationship through streamlines at the end of the first time step, after 6 months.

Reservoir properties distribution of porosity and permeability are implemented in such way that a non-homogeneous synthetic reservoir is created. To examine the effect of aquifer in multi-objective GA optimization of injection efficiencies a strong aquifer is defined and connected to the top and right sides of reservoir. Producers liquid rate and total injectors water rate target are listed in the Table 3. Initial water injection rate of $500 \mathrm{Stb} /$ day is executed for each injector in the conventional streamline simulation optimization method for 6 years.

\section{Results and discussion}

In order to optimize the injection rate portions, genetic algorithm coupled with streamline simulation has been implemented in each timestep. The Pareto front solution set is produced in evaluation of the best answer.

Figure 3 shows the Pareto front solution set for scenario $E$ in the second timestep of the represented synthetic model. Non-optimized injection rates are shown in red and the optimized ones are in blue. Ultimately, a point which has relatively higher oil production with lower water production and lower water loss was chosen from the Pareto front solution set as the best answer.

Figure 4 represents the comparison of field cumulative oil and water production and water loss to the aquifer of different scenarios in the second time step. Scenario E
Fig. 2 Schematic of synthetic model
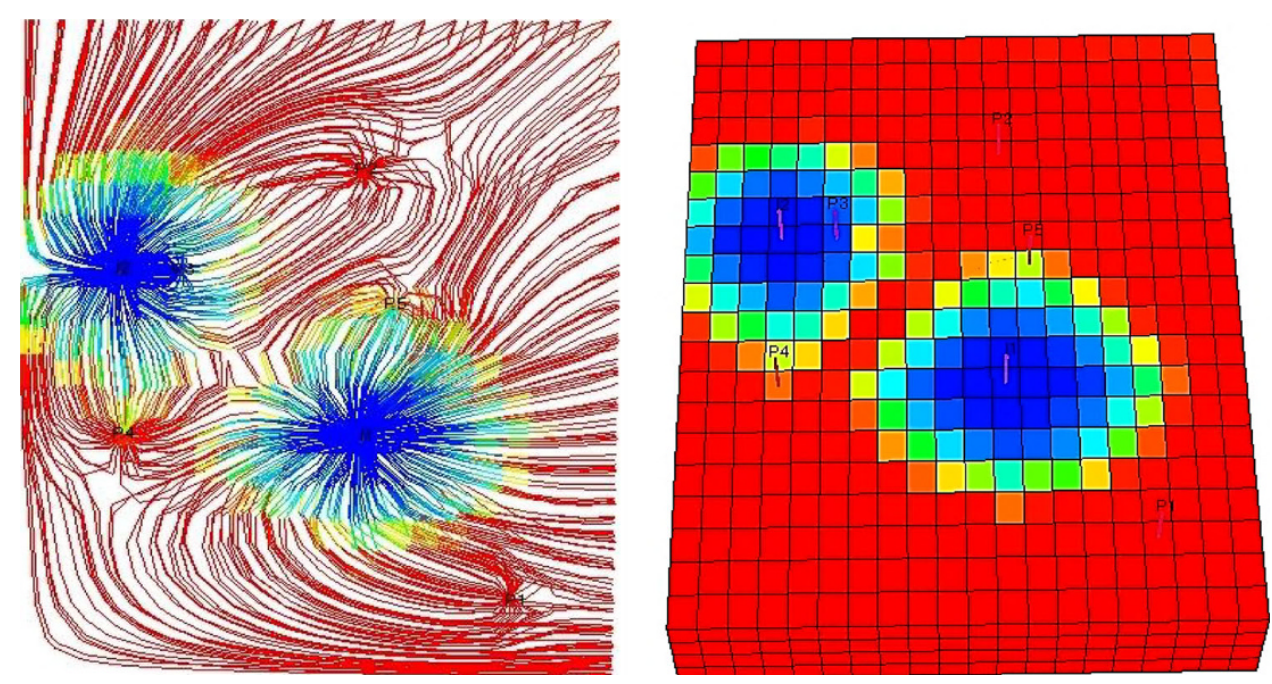
Table 3 Production and injection control schedule

\begin{tabular}{lc}
\hline Well name & Liquid rate (Stb/day) \\
\hline $\mathrm{P}_{1}$ & 75 \\
$\mathrm{P}_{2}$ & 100 \\
$\mathrm{P}_{3}$ & 100 \\
$\mathrm{P}_{4}$ & 125 \\
$\mathrm{P}_{5}$ & 90 \\
$\mathrm{I}_{1}+\mathrm{I}_{2}$ & 1,000 \\
\hline
\end{tabular}

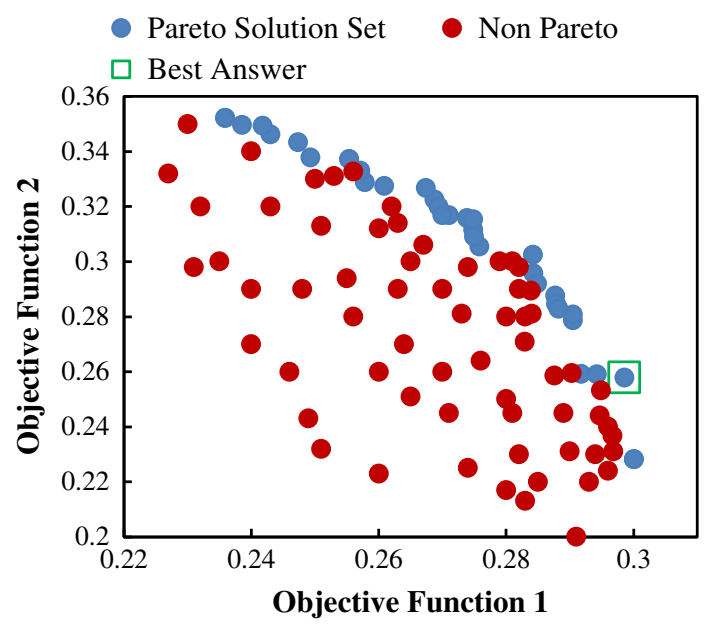

Fig. 3 Pareto-optimal set solution and making the best decision in scenario $\mathrm{E}$

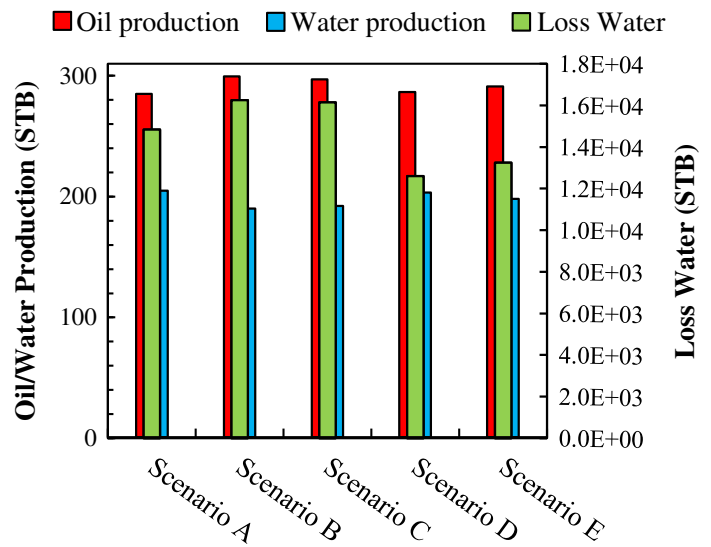

Fig. 4 Comparison of field oil, water production and water loss of different scenarios in the second timestep

causes a decrease in water loss of about $20 \%$, while it has not an undesirable effect on oil and water production. The highest oil production and the lowest water production were obtained in the scenario B which maximizes the total oil production. It may lead to reach the maximum economic benefit from the field after comprehensive assessment.

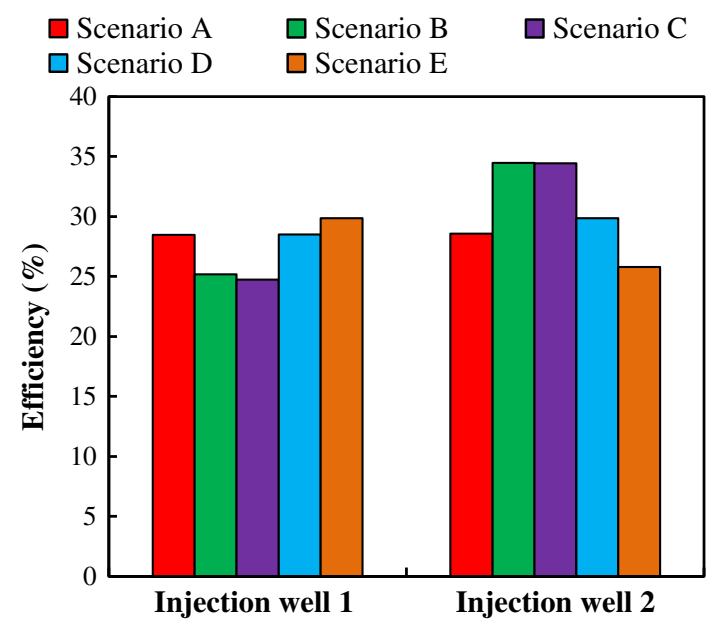

Fig. 5 Comparison of injection efficiencies of different MOGA scenarios in the second timestep

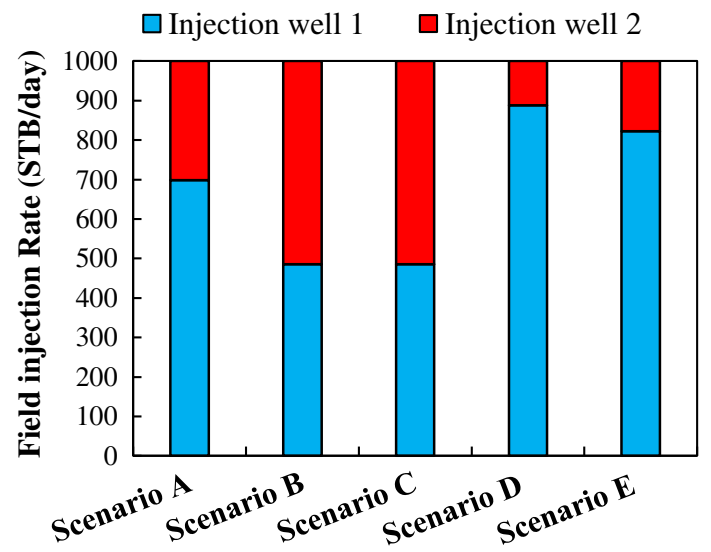

Fig. 6 Allocation of injected water in MOGA scenarios in the second timestep

Injection efficiencies of the injectors for the second timestep are illustrated in Fig. 5. Although average injection efficiency of $30 \%$ in scenario B is more than other scenarios, optimization implementation regardless of production limitation and aquifer performance (similar to the conventional methods) is not recommended. Therefore, it is not unexpected that scenario B has a higher water loss than other scenarios. Furthermore, it reveals that there is no considerable difference in field average pressure in different scenarios.

Optimized injection rates of different MOGA scenarios are shown in Fig. 6 for the second timestep. The maximum oil production can be obtained by selecting the injection rate of 485 and $515 \mathrm{Stb} /$ day for the well 1 and well 2, respectively, while to reduce the amount of losing water to the aquifer and minimizing the operational cost simultaneously, the injection rate of 888 and $112 \mathrm{Stb} /$ day should be chosen for the following wells. 

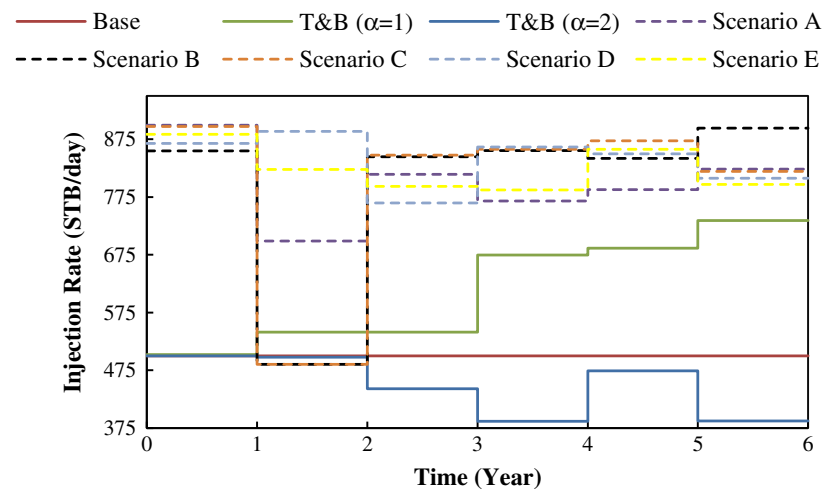

Fig. 7 optimized injection rate in well 1 during 6-year simulation in MOGA scenarios and conventional methods
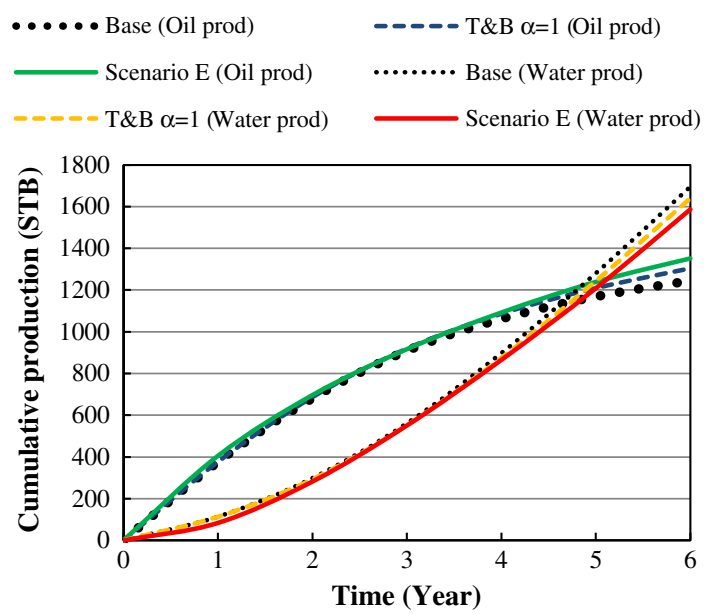

Fig. 8 Cumulative production of oil and water for scenario E and conventional methods

\section{Six-year optimization}

A comparison with previous approach has been established to evaluate the advantages of the presented method in this paper. Therefore, in addition to the baseline constant injection rate, two simulation runs with $\alpha=1$ and $\alpha=2$ have been executed by Thiele and Batycky approach. In their approach, the best injection rate of the next step was chosen with respect to the field injection efficiency of the current step. Here to obtain the best answer in each timestep, the automated injection rate optimization runs were repeated 30 times. Similar to the other conventional optimization methods, one of the disadvantages of this methodology is that it may converge on the local solution instead of global ones. The other weakness is the impression of the previous timestep in the current timestep solution. On the other hand, using the best answer as a global solution in each timestep of optimization is the unique feature of all MOGA scenarios. Figure 7 shows the

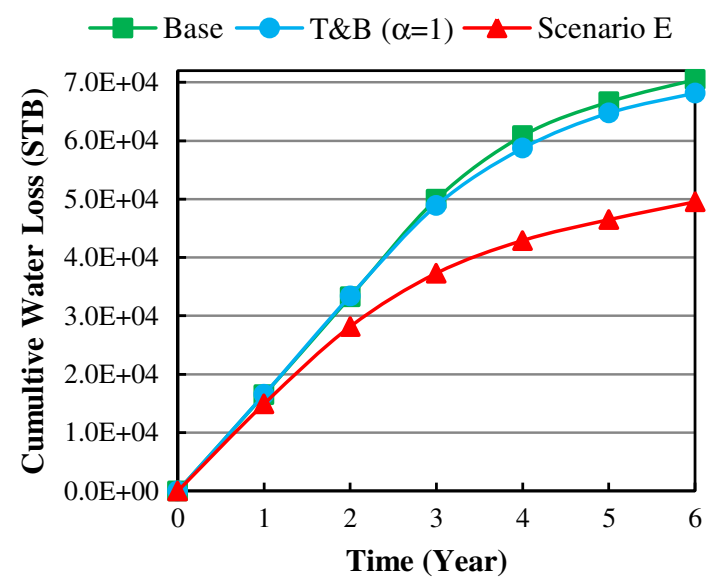

Fig. 9 Reduction of water loss for MOGA scenario E compared with conventional methods

optimized injection rate in well 1 in different methods during the 6-year simulation. While Thiele and Batycky approach with $\alpha=2$ tends to reduce the injection rate of well 1 , using this approach with $\alpha=1$ increases the amount of injection rate. In general, all the MOGA scenarios have much more better results than other conventional methods.

From all of MOGA scenarios, scenario E was selected to compare with conventional approaches because of applying most operational limitations in its optimization process. Figure 8 shows the cumulative production of oil and water for 6-year simulation. In MOGA scenario E optimization, the highest oil production with the lowest water production has been observed in comparison with other methods.

In the Thiele and Batycky approach there is no considerable control on decreasing water loss to the aquifer, even though the observed reduction in Fig. 9 by this approach is negligible. However, in MOGA scenario E besides increasing in oil production, significant decline in water loss to the aquifer is observed in Fig. 9.

Evaluation of the injection efficiencies and injection rates in different wells and scenarios in is shown in Figs. 10, 11. Although injection efficiencies are almost the same in different scenarios and different wells, by allocating the injection rates in this paper, higher field performance is achieved.

Cumulative oil and water production and loss of water for 6-year production of all optimization methods are shown in Fig. 12.

In the best case of Thiele and Batycky approach with $\alpha=1$, ultimate oil recovery improves up to $4.7 \%$ in comparison with baseline, while using various MOGAs at the worst condition cause to increase in ultimate oil production about $7.3 \%$ and in the best case, it increases it about $11 \%$. Besides, using any MOGAs decrease the total water production. Thiele and Batycky approach with $\alpha=2$ 


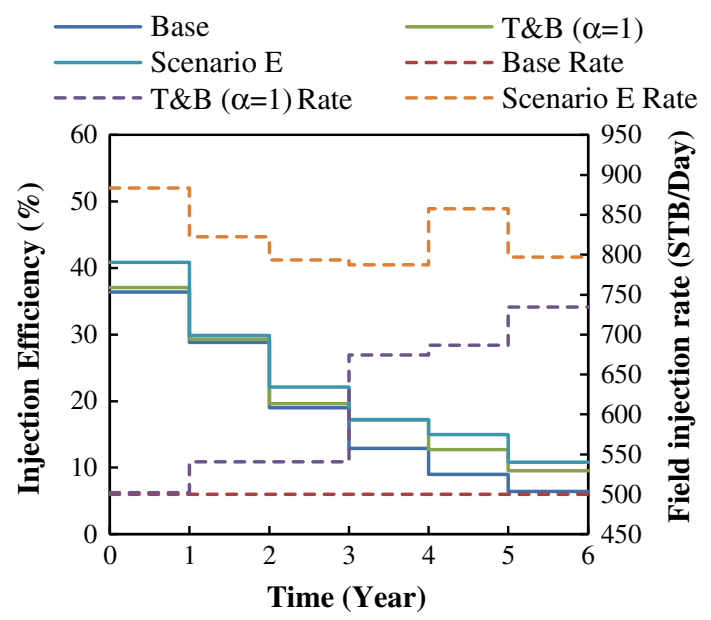

Fig. 10 Optimized injection efficiency and injection rate for MOGA scenarios and conventional method in well 1

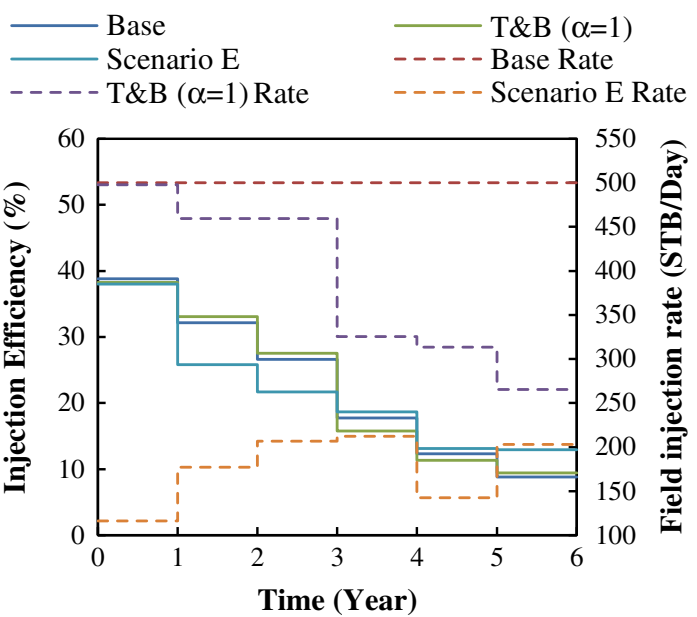

Fig. 11 Optimized injection efficiency and injection rate for MOGA scenarios and conventional method in well 2

results in rise in water loss to the aquifer and with $\alpha=1$, it can only reduce water loss about $3 \%$. Multi-objective genetic algorithm scenarios can decrease the amount of water loss about $23-32 \%$.

\section{Conclusion}

Implementing the multi-objective genetic algorithm always offers the best solution duo to its fundamentals and characteristic. Comparison of the proposed approach in this paper with conventional workflow shows that all MOGA scenarios have better proficiencies.

- The primary objective of this work has achieved by using multi-objective algorithm genetic to conclude higher injection efficiency instead of conventional methods.

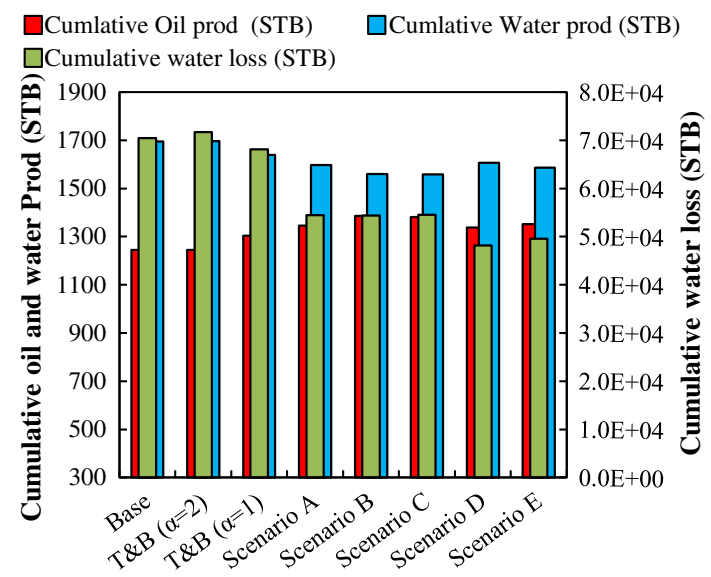

Fig. 12 Total oil and water production in different scenarios

- To improve the weakness of the other methods, secondary objectives have been defined as various scenarios which cause higher total oil production, lower water production or less water loss to the aquifer.

- Pareto front shows all the answer sets in each time step that implementing different scenarios help to select the best one with different consideration aspects.

- Streamline simulation has been used to produce the allocated offset oil production in injectors and has been improved in each time step by the means of multiobjective genetic algorithm.

Open Access This article is distributed under the terms of the Creative Commons Attribution License which permits any use, distribution, and reproduction in any medium, provided the original author(s) and the source are credited.

\section{Appendix}

Thiele and Batycky workflow injection efficiency optimization:

1. Determining injection efficiencies at the current time

2. Re-allocation of water injection with respect to the to the average field efficiency,

$$
\begin{aligned}
& w_{i}=\min \left[w_{\max }, w_{\max }\left(\frac{e_{i}-\bar{e}}{e_{\max }-\bar{e}}\right)^{\alpha}\right], \text { if } e_{i}>\bar{e} \\
& w_{i}=\max \left[w_{\min }, w_{\min }\left(\frac{\bar{e}-e_{i}}{\bar{e}-e_{\min }}\right)^{\alpha}\right], \text { if } e_{i}<\bar{e}
\end{aligned}
$$

$e_{i}$ : Injection efficiency

$\bar{e}$ : Average field injection efficiency

$w_{\max }$ : Maximum weight at $e_{\max }$

$w_{\min }$ : Minimum weight at $e_{\min }$ 
$e_{\max }:$ Upper injection efficiency limit

$e_{\text {min }}$ : Lower injection efficiency limit

3. Determining new injection rates

$q_{i}^{\text {new }}=w_{i} \times q_{i}^{\text {old }}$

4. Normalizing new injection rates and determining the production rates using streamline simulation (Iino and Arihara 2007; Thiele et al. 2003).

\section{References}

Alhuthali A, Oyerinde A, Datta-Gupta A (2007) Optimal waterflood management using rate control, Society of Petroleum Engineers, SPE-102478-PA

Konak A, Coit DW, Smith AE (2006) Multi-objective optimization using genetic algorithms: a tutorial. Reliab Eng Syst Saf 91:992-1007. doi:10.1016/j.ress.2005.11.018

Iino A, Arihara N (2007) Use of streamline simulation for waterflood management in naturally fractured reservoirs, SPE 108685. In: International oil conference and exhibition. Mexico
Marler RT, Arora JS (2004) Survey of multi-objective optimization methods for engineering. Struct Multidisc Optim 26:369-395. doi:10.1007/s00158-003-0368-6

Samier P, Quettier L, Thiele M (2001) Applications of streamline simulations to reservoir studies. In: SPE reservoir simulation symposium. Texas, USA

Sbalzariniy IF, Sibylle M, Koumoutsakosyz P (2000) Multiobjective optimization using evolutionary algorithms, Center for turbulence research

Thiele MR (2001) Streamline Simulation. In: 6th international forum on reservoir simulation, Austria

Thiele MR, Batycky RP, Blunt MJ (1997) Streamline-based 3d fieldscale compositional reservoir simulator, SPE 38889. In: SPE annual technical conference and exhibition. Texas, USA

Thiele MR, Batycky RP, Streamsim Technologies (2003) Water injection optimization using a streamline-based workflow, SPE 84080. In: SPE annual technical conference and exhibition. Colorado, USA

Xie Q, Li S, Yang G (2005) Studies on fast pareto genetic algorithm based on fast fitness identification and external population updating scheme, Chinese academy of science 